\title{
Rapid Authentication of Extra Virgin Olive Oil using UV and FTIR Spectroscopy
}

\author{
H. Hashem ${ }^{1}$, Rania I.M. Almoselhy ${ }^{2}$, M. El-Waseif ${ }^{1}$ and A. Magdy ${ }^{1}$
}

${ }^{I}$ Food Science and Technology Department, Faculty of Agriculture, Al-Azhar University, Cairo, Egypt. ${ }^{2}$ Oils and Fats Research Department, Food Technology Research Institute, Agricultural Research Center, Giza, Egypt.

Received: 24 Jan. 2020 / Accepted 30 Mar. 2020 / Publication date: 30 April 2020

\begin{abstract}
Spectroscopic examination in the ultraviolet (UV) can provide information on the quality of an oil, its state of preservation and changes brought about by technological processes. The absorption at the wavelengths $232 \mathrm{~nm}$ and $270 \mathrm{~nm}$ in cyclohexane is due to the presence of conjugated diene and triene systems, respectively, resulting from oxidation processes and/or refining practices. These absorptions are expressed as specific extinctions conventionally indicated by $\mathrm{K}$ (also referred to as "extinction coefficient"). In this work, the specific extinctions at $232 \mathrm{~nm}$ and $270 \mathrm{~nm}$ in cyclohexane are calculated for a concentration of $1 \% \mathrm{w} / \mathrm{v}$ for extra virgin olive oil adulteration with sunflower, corn oils in their binary admixtures in different concentrations $(0,5,15,25$ and $100 \% \mathrm{w} / \mathrm{w})$. Results revealed that the adulteration will be detected easily by UV absorbance at $270 \mathrm{~nm}$ because sunflower oil showed a shift out of the permitted range at $15 \%$ upward higher concentrations added to extra virgin olive oil. Also, corn oil will be detected if present in $15 \%$ upward higher concentrations in blends with extra virgin olive oil. The calculated $\Delta \mathrm{K}$ allowed the detection of adulteration in extra virgin olive oil at the concentration of $15 \%$ upward higher concentrations of adulterant sunflower and corn oils; and this is the lowest detectable value in adulterated extra virgin olive oil. Also, Fourier Transform Infrared Spectroscopy (FTIR) is used as an effective analytical tool in order to determine extra virgin olive oil adulteration with sunflower, corn oils in their binary admixtures in different concentrations $(0,5,15$, 25 and $100 \% \mathrm{w} / \mathrm{w}$ ). A band around $2925 \mathrm{~cm}^{-1}$ along with its intensity (assigned to $\mathrm{C}-\mathrm{O}$ stretching and symmetric stretching vibration of the aliphatic $\mathrm{CH}_{2}$ group) along with a very strong band along with its intensity located at $1743 \mathrm{~cm}^{-1}$ (assigned to the $\mathrm{C}=\mathrm{O}$ stretching band of the ester group) were important to detect concentration of 5\% adulterants $\mathrm{SO}$ and $\mathrm{CO}$ in EVOO. The present work successfully achieved its aim included in the applications of UV and FTIR spectroscopy as rapid, cheap, nondestructive, authenticity measuring tools to assess the adulteration of extra virgin olive oil with other edible oils such as corn and sunflower oils in detection limit 15\% for UV and 5\% for FTIR spectroscopy. Library searching in the FTIR region is a well established and powerful way which was used in comparison and matching of measured spectra.
\end{abstract}

Keywords: Authentication; UV; FTIR; Spectroscopy; Extra virgin olive oil; Sunflower oil; Corn oil

\section{Introduction}

Over the last decades, olive oil quality and authenticity control has become an issue of great importance to consumers, suppliers, retailers, and regulators in both traditional and emerging olive oil producing countries, mainly due to the increasing worldwide popularity and the trade globalization of this product. Thus, in order to ensure olive oil authentication, various national and international laws and regulations have been adopted (Bajoub et al., 2018). Olive oil sector has faced a new and growing boost aimed at the conversion of the traditional extraction process to a new and highly automated one. Innovative technologies are experimentally applied to the virgin olive oil extraction process in order to make it continuous and more efficient (Caponio et al., 2019).

Extra virgin olive oil (EVOO) has unique nutritional and sensory characteristics. The importance of EVOO is mainly attributed to its high content of oleic acid and its richness in phenolic compounds, which act as natural antioxidants. On the other hand, EVOO is expensive owing to the hard and timeconsuming tasks involved in the cultivation of olive trees, the harvesting of the fruits, and the extraction of the oil. For these reasons, adulterations of EVOO with olive oils of lower quality are occasionally detected (Lerma-García et al., 2010).

Corresponding Author: A. Magdy, Food Science and Technology Department, Faculty of Agriculture, Al-Azhar University, Cairo, Egypt. E-mail: adelmagdey26@gmail.com 
EVOO has high resistance to oxidation as well as advantageous frying properties when compared to other vegetable oils due to its fatty acid composition; high monounsaturated fatty acids (55-88\%), low polyunsaturated fatty acids (2-21\%); and the presence of highly antioxidative phenolic compounds that inhibit the production of hydroperoxides (Gonçalves et al., 2014).

Recently, a chemopreventive activity of olive oil has been attributed to its unique phenolic compounds represented by phenolic alcohols like hydroxytyrosol and tyrosol, and their secoiridoid derivatives (oleuropein aglycon, ligstroside aglycon, oleocanthal), and oleuropein. Olive oil can also protect against carcinogenic agents that are abundant in the environment and food. High content of antioxidant polyphenols (hydroxytyrosol, oleuropein) makes virgin olive oil relatively stable and resistant to oxidation, and thus minimizing the risk of formation of potentially carcinogenic products of lipid peroxidation upon storage (Monika et al., 2018).

Determination of authenticity of EVOO has become very important in recent years due to the increasing public concerns about possible adulterations with relatively cheap vegetable oils such as sunflower, soybean, sesame, corn and refined olive oils (Almoselhy Rania et al., 2009). Olive oil is one of the oil components used in some pharmaceutical preparations such as cream and lotion, therefore its authenticity is very important to assure the quality of pharmaceutical products. The diversity of oil adulterants on the market has increased demand for analytical techniques needed to verify the authenticity of EVOO (Nurwahidah et al., 2019).

The adulteration practice in oils is difficult to detect, when the oil adulterant has close similarity in terms of chemical composition and color to that of the original oil. The development of a fast and reliable technique for qualitative and quantitative analyses of high value oils like EVOO is one of the interest aspects for researchers. Due to their properties as fingerprint techniques, vibrational spectroscopies like infrared and Raman have been considered deal analytical methods for food studies, including the authenticity of high price edible fats and oils (Rohman et al., 2014).

Spectrophotometric examination in the UV can provide information on the quality of an oil, its state of preservation and changes brought about by technological processes. The absorption at the wavelengths specified in the method is due to the presence of conjugated diene and triene systems resulting from oxidation processes and/or refining practices (IOC, 2019a).

Many efforts have been made in order to correlate the chemical structures of naturally occurring molecules in EVOO with its quality and genuineness, health benefits and sensory characteristics. The study of the different molecules present in EVOO has required the use of different sample preparation methodologies and analytical techniques. Among these techniques can be listed spectrometric techniques. FTIR spectroscopy was found to be the most efficient in classification of oils and fats (Gómez-Caravaca et al., 2016). FTIR applications were strongly used more than 10 years ago due to its simplicity, sensitivity and rapidness (AlHanash et al., 2018).

The present work aimed at the applications of UV and FTIR spectroscopy to assess the adulteration of extra virgin olive oil with sunflower and corn oils.

\section{Materials and Methods}

\section{Materials:}

Extra virgin olive oil was extracted from fresh olive fruits by Oliomio-machine then filtered and kept in brown glass bottles at $-5^{\circ} \mathrm{C}$. Sunflower oil and corn oil samples were purchased locally. According to their labels, all the oils used in adulteration were additive-free, refined, bleached and deodorized (RBD). Solvents and chemicals used were all of analytical grades.

\section{Analytical Methods:}

Different admixtures of various concentrations $(0,5,15,25$ and 100\%) of the above used edible oils were prepared $(\mathrm{w} / \mathrm{w})$ as binary mixtures with extra virgin olive oil.

\section{Ultraviolet (UV) Spectroscopic Characteristics:}

Natural conjugated constituents are determined by measuring UV absorption at specific wavelengths in purified solvent. 
The ultraviolet spectra of organic compounds are attributable to electronic excitations or transitions. Functional groups with high electron densities such as carbonyl groups, nitro groups, double, triple bonds and conjugated double bonds are usually considered. The sample was dissolved in freshly distilled (95\%) ethanol to a known concentration. The absorption was then read at $232 \mathrm{~nm}$ for conjugated diene, and at $270 \mathrm{~nm}$ for conjugated triene in quartz cell with $1 \mathrm{~cm}$ path length against a solvent blank in a matching cell using a PyeUnicam double-beam spectrophotometer, model SP1800, as described by Kates (1972), Absorptivity for conjugated constituents was calculated, applying the following relationship:

where; $\mathrm{A}=$ observed absorbance

$\mathrm{b}=$ cell length in $\mathrm{cm}$

$\mathrm{c}=\mathrm{g}$ test portion / $\mathrm{L}$ final dilution used for $\mathrm{K}$ measurement

The $\Delta \mathrm{K}$ value was calculated according to IOC (2019a) as following:

$\Delta \mathrm{K}=\mathrm{K}_{270}-\left[\left(\mathrm{K}_{266}+\mathrm{K}_{274}\right) / 2\right]$

\section{FTIR Spectroscopic Characteristics:}

FTIR spectral analysis was carried out according to the method outlined by Guillén and Cabo (1999) as following:

A number of 9 sets of extra virgin olive oil mixed with each of the adulterant oils; sunflower, corn oils in different proportions $(5,15,25$ and $100 \%)$ on $\mathrm{w} / \mathrm{w}$ basis without sample preparation procedures were exposed to FTIR analysis.

\section{Instrumentation and FTIR Spectral Data Acquisition:}

A Jasco Spectrum FT-IR Plus 460 Spectrophotometer (Japan) equipped with a deuterated triglycerine sulphate (DTGS) detector was used to obtain FT-IR spectra. $5 \mu 1$ of the sample was pressed between two well-polished $\mathrm{KBr}$ disks (liquid cell) creating a thin film. Each sample undergoes 64 scans which are accumulated in one measurement to acquire a sufficient signal-to-noise ratio. Samples were scanned between 4000 and $400 \mathrm{~cm}^{-1}$ with a nominal resolution of $4 \mathrm{~cm}^{-1}$ and the data interval was $1 \mathrm{~cm}^{-1}$ (Allam and Hamed, 2007).

\section{Results and Discussion}

\section{Ultraviolet (UV) Spectroscopic Characteristics:}

The spectrophotometric measurement is one of the physical methods which is particularly useful in composition studies on fats and oils and in the analysis and identification of fatty materials. It had been employed for studying and following chemical reactions of fatty materials, especially isomerization, polymerization, oxidation and the determination of rancidity in oils. UV spectrophotometry offers an accurate, rapid, and economically viable testing method to determine the purity of extra virgin olive oils. Unsaturated fatty materials absorb light in the ultraviolet region of the spectrum; this region is between about 200 and $400 \mathrm{~nm}$. In the case of non-conjugated and saturated materials, the absorption is weak and cannot be used for analytical purposes. When linoleic and linolenic acid or more highly unsaturated fatty acids are oxidized to form hydroperoxides, the double bonds in the oils become conjugated. The linoleic and linolenic acids are isomerized to conjugated dienoic and trienoic acids, respectively. The hydroperoxides and the conjugated diene which may result from its decomposition show a strong absorption band at about $232 \mathrm{~nm}$, while conjugated triene and the secondary oxidation products, particularly diketones, show an absorption band at $270 \mathrm{~nm}$.

According to the regulations and recommendations stated in the official Commission Implementing Regulation (EU) No 1348/2013 of 16 December 2013 amending Regulation (EEC) No $2568 / 91$ on the characteristics of olive oil and olive-residue oil and on the relevant methods of analysis (2013), the extinction coefficients $\mathrm{K}_{232}$ and $\mathrm{K}_{270}$ for extra virgin olive oil (EVOO) should be $\leq 2.5$ and $\leq 0.22$, respectively and $\Delta \mathrm{K}$ should be $\leq 0.01$.

Conjugated diene absorbencies at $232 \mathrm{~nm}$, conjugated triene absorbencies at $270 \mathrm{~nm}$ and $\Delta \mathrm{K}$ of extra virgin olive oil in binary admixtures with the different adulterant oils (sunflower and corn oils) at various concentrations of adulterant oils are listed in Tables (1,2 and 3), respectively. 
Table 1: Conjugated diene absorbencies (at $232 \mathrm{~nm}$ ) of EVOO in binary admixtures with different adulterant oils ( $\mathrm{SO}$ and $\mathrm{CO}$ ) at various concentrations

Adulterant oil

\begin{tabular}{cll}
\cline { 2 - 3 }$\%$ & EVOO + SO & EVOO + CO \\
\hline $\mathbf{0}$ & $1.736(\mathrm{EVOO})$ & $1.736(\mathrm{EVOO})$ \\
$\mathbf{5}$ & 2.032 & 1.654 \\
$\mathbf{1 5}$ & 2.472 & 2.385 \\
$\mathbf{2 5}$ & 2.927 & 2.529 \\
$\mathbf{1 0 0}$ & $1.916(\mathrm{SO})$ & $2.032(\mathrm{CO})$ \\
\hline
\end{tabular}

EVOO; extra virgin olive oil $\quad \mathrm{SO}$; sunflower oil $\mathrm{CO}$; corn oil

Table 2: Conjugated triene absorbencies (at $270 \mathrm{~nm}$ ) of EVOO in binary admixtures with different adulterant oils ( $\mathrm{SO}$ and $\mathrm{CO}$ ) at various concentrations

\begin{tabular}{cll}
$\begin{array}{c}\text { Adulterant oil } \\
\%\end{array}$ & & \\
\cline { 2 - 3 } & EVOO+ SO & EVOO+ CO \\
\hline $\mathbf{0}$ & $0.149(\mathrm{EVOO})$ & $0.149(\mathrm{EVOO})$ \\
$\mathbf{5}$ & 0.212 & 0.221 \\
$\mathbf{1 5}$ & 0.552 & 0.367 \\
$\mathbf{2 5}$ & 0.651 & 0.383 \\
$\mathbf{1 0 0}$ & $1.53(\mathrm{SO})$ & $1.707(\mathrm{CO})$ \\
\hline
\end{tabular}

EVOO; extra virgin olive oil $\quad$ SO; sunflower oil $\quad$ CO; corn oil

Table 3: Determination of $\Delta \mathrm{K}$ of EVOO in binary admixtures with different adulterant oils ( $\mathrm{SO}$ and $\mathrm{CO})$ at various concentrations

\begin{tabular}{cll}
\hline $\begin{array}{c}\text { Adulterant oil } \\
\text { \% }\end{array}$ & EVOO + SO & \multicolumn{1}{c}{ E } \\
\cline { 2 - 3 } $\mathbf{0}(\mathbf{E V O O})$ & $-0.002(\mathrm{EVOO})$ & $-0.002(\mathrm{EVOO})$ \\
$\mathbf{5}$ & 0.0025 & 0.005 \\
$\mathbf{1 5}$ & 0.028 & 0.012 \\
$\mathbf{2 5}$ & 0.033 & 0.015 \\
$\mathbf{1 0 0}$ (SO) & $0.118(\mathrm{SO})$ & $0.1025(\mathrm{CO})$ \\
\hline
\end{tabular}

EVOO; extra virgin olive oil $\quad \mathrm{SO}$; sunflower oil $\quad \mathrm{CO}$; corn oil

From Table (1), it appears that $\mathrm{K}_{232}$ has the value of 1.736 for EVOO which agrees with Codex Alimentarius Standard (2013); IOC (2019b). From the same table, it is noticed that the values of $\mathrm{K}_{232}$ for pure sunflower and corn oils were 1.916 and 2.032, respectively. Also, the values of $\mathrm{K}_{232}$ for the binary admixtures of adulterant oils with EVOO at concentrations $25 \%$ of adulterant $\mathrm{SO}$ and $\mathrm{CO}$ were 2.927 and 2.529, respectively, which are outside the limits of Codex Alimentarius Standard (2013); IOC (2019b).

From Table (2), it appears that $\mathrm{K}_{270}$ has the value of 0.149 for EVOO which agrees with Codex Alimentarius Standard (2013); IOC (2019b). From the same table, it is noticed that the values of $\mathrm{K}_{270}$ for pure sunflower and corn oils were 1.53 and 1.707, respectively. Also, the values of $\mathrm{K}_{270}$ for the binary admixtures of adulterant oils with $\mathrm{EVOO}$ at concentrations $15 \%$ of adulterant $\mathrm{SO}$ and $\mathrm{CO}$ were 0.552 and 0.367 , whereas, at concentrations $25 \%$ of adulterant SO and $\mathrm{CO}$ were 0.651 and 0.383 , respectively, which are outside the limits of Codex Alimentarius Standard (2013); IOC (2019b).From Table (3), it appears that $\Delta \mathrm{K}$ has the value of -0.002 for EVOO which agrees with Codex Alimentarius Standard (2013); IOC (2019b). From the same table, it is noticed that the values of $\Delta \mathrm{K}$ for pure sunflower and corn oils were 0.118 and 0.1025 , respectively. Also, the values of $\Delta \mathrm{K}$ for the binary admixtures of adulterant oils with EVOO at concentrations $15 \%$ of adulterant $\mathrm{SO}$ and $\mathrm{CO}$ were 0.028 and 0.012 , whereas, at concentrations $25 \%$ of adulterant $\mathrm{SO}$ and $\mathrm{CO}$ were 0.033 and 0.015 , respectively, which are outside the limits of Codex Alimentarius Standard (2013); IOC (2019b).

From the above results, it appears the small value in absorbency at $270 \mathrm{~nm}$ of extra virgin olive oil might be further contributed to its low concentration of linoleic acid coupled with surplus oxidative 
stability of this oil, and subsequently low relative rates of formation of ketonic and aldehydic oxidative products, as well as low formation of conjugated trienes. While, the more absorption at $270 \mathrm{~nm}$ caused by the other studied oils which exceeded that of extra virgin olive oil, might be attributed to their higher oxidative degradation of the polyunsaturated linoleate present in these oils at higher concentrations resulting in the formation of conjugated trienoic and ketodienes. The excess UV absorbance could be also explained by the higher amount of activated methylene groups in polyunsaturated fatty acids in these oils than in extra virgin olive oil which are oxidized to form secondary oxidative products.

The obtained results in Table (2) revealed that the UV absorbency at $270 \mathrm{~nm}$ of EVOO gradually increased with stepping up the concentration of the different adulterant oils in its binary admixtures. It was clearly noticed that the adulteration with adulterant $\mathrm{SO}$ and $\mathrm{CO}$ will be detected easily by UV absorbency at $270 \mathrm{~nm}$ because it showed a shift out of the range of Codex Alimentarius Standard (2013); IOC (2019b) at 15\% upward its higher concentrations with EVOO.

$\Delta \mathrm{K}$ is useful for readily classifying olive oil quality according to the following value: $\Delta \mathrm{K}$ for extra virgin olive oil $\leq 0.01$ as stipulated by Codex Alimentarius Standard (2013); IOC (2019b).

$\Delta \mathrm{K}$ values of extra virgin olive oil in binary admixtures with adulterant studied oils at various concentrations were tabulated in Table (3). It is apparent that $\Delta \mathrm{K}$ had the value of -0.002 for extra virgin olive oil which agree with the aforementioned permitted level stipulated by Codex Alimentarius Standard (2013); IOC (2019b). The values of $\Delta \mathrm{K}$ for pure sunflower and corn oils were 0.118 and 0.1025 , respectively, being higher than that of extra virgin olive oil.

Concerning the adulteration of extra virgin olive oil with the adulterant sunflower oil, $\Delta \mathrm{K}$ values of binary admixtures of extra virgin olive oil with this oil are shown in Table (3) indicating that the $\Delta \mathrm{K}$ values increased gradually with increasing the concentration of the adulterant sunflower oil in the blends ranging from -0.002 for extra virgin olive oil, till pure sunflower oil. Fortunately, it was found that $\Delta \mathrm{K}$ at $15 \%$ adulterant sunflower oil in binary admixture with extra virgin olive oil was 0.028 which made the blend out of the level of Codex Alimentarius Standard (2013); IOC (2019b), which implies that $\Delta \mathrm{K} \leq 0.01$ for extra virgin olive oil. These findings mean that it is possible to detect the adulterant sunflower oil at $15 \%$ in its binary admixture with extra virgin olive oil.

Similarly, from $\Delta \mathrm{K}$ values of the binary admixtures of extra virgin olive oil with the adulterant corn oil shown in Table (3), $\Delta \mathrm{K}$ increased gradually with increasing concentration of the adulterant corn oil in the blends ranging from -0.002 for extra virgin olive oil, till pure corn oil. It was found that the $\Delta \mathrm{K}$ at $15 \%$ adulterant corn oil in binary admixture with extra virgin olive oil was 0.012 which made the blend out of the level of Codex Alimentarius Standard (2013); IOC (2019b), which implies that $\Delta \mathrm{K}$ $\leq 0.01$ for extra virgin olive oil. Hereupon, it is possible to detect the adulterant corn oil at $15 \%$ in its binary admixture with extra virgin olive oil.

In conclusion, adulteration of extra virgin olive oil with adulterant sunflower and corn oils could be detected at $15 \%$ of adulterant oil added; using $\Delta \mathrm{K}$ values obtained from $\mathrm{UV}$ characteristics, and this is the lowest detectable value in adulterated extra virgin olive oil.

\section{FTIR Spectroscopic Characteristics:}

According to Guillén and Cabo (1999); the presence of small amounts of seed oil (sunflower, corn, walnut, rapeseed, soybean, safflower, peanut, wheat germ and sesame oil) in olive oil is shown by a small variation in the values of the frequencies of specific bands of the spectra, resulting from a smaller proportion of monounsaturated acyl groups than in pure olive oil.

In the present study, FTIR spectra of pure oils (extra virgin olive oil, sunflower oil and corn oil), together with the spectra of different blends of extra virgin olive oil with both (sunflower, corn oils) are shown in Figures (1-9) and summarized in Tables (4, 5 and 6).

It is difficult to differentiate between these oils by just visual examination of their whole spectrum. However, a careful investigation of the spectral regions revealed that there are visual differences in the absorption intensity around $1163 \mathrm{~cm}^{-1}$ (assigned to $-\mathrm{C}-\mathrm{O}$ stretching and $-\mathrm{CH}_{2}$ bending). EVOO has absorption intensity at $1160.94 \mathrm{~cm}^{-1}$, whereas, SO has absorption intensity at $1164.79 \mathrm{~cm}^{-1}$ and $\mathrm{CO}$ has absorption intensity at $1157.08 \mathrm{~cm}^{-1}$. 
Table 4: FTIR spectral data of pure oils (extra virgin olive oil, sunflower oil, corn oil)

\begin{tabular}{|c|c|c|c|c|c|}
\hline \multicolumn{2}{|c|}{ EVOO 100\% } & \multicolumn{2}{|c|}{ SO 100\% } & \multicolumn{2}{|c|}{ CO 100\% } \\
\hline $\mathbf{c m}^{-1}$ & $\% \mathrm{~T}$ & $\mathbf{c m}^{-1}$ & $\% \mathrm{~T}$ & $\mathbf{c m}^{-1}$ & $\% \mathrm{~T}$ \\
\hline 3467.38 & 96.97 & 3457.74 & 92.65 & 3445.21 & 94.84 \\
\hline 2921.63 & 91.13 & 2922.59 & 96.04 & 2921.63 & 94.45 \\
\hline 2865.06 & 92.53 & 2855.1 & 96.63 & 2855.1 & 94.92 \\
\hline 2368.16 & 102.92 & 2376.84 & 98.80 & 2373.94 & 96.38 \\
\hline 2039.35 & 102.95 & & & & \\
\hline 1743.33 & 92.30 & 1740.44 & 95.54 & 1741.41 & 94.41 \\
\hline 1654.62 & 100.30 & 1640.16 & 95.40 & 1643.05 & 95.57 \\
\hline 1454.06 & 94.40 & 1450.21 & 95.84 & 1451.17 & 95.33 \\
\hline 1367.28 & 95.72 & 1376.93 & 95.88 & & \\
\hline 1234.22 & 94.65 & & & & \\
\hline 1160.94 & 93.02 & 1164.79 & 95.48 & 1157.08 & 95.12 \\
\hline & & 1021.12 & 96.37 & & \\
\hline 719.318 & 96.71 & & & 712.56 & 96.54 \\
\hline 596.861 & 101.82 & 609.39 & 95.82 & & \\
\hline & & 434.869 & 97.71 & & \\
\hline
\end{tabular}

A peak ascribed to the very weak stretching of the $\mathrm{C}=\mathrm{O}$ of the acid group located at 1654.62 $\mathrm{cm}^{-1}$ appears as a shoulder of the very strong band of the $\mathrm{C}=\mathrm{O}$ stretching band of the ester group located at $1743.33 \mathrm{~cm}^{-1}$ in EVOO. These two peaks were observed at lower wavelengths in $\mathrm{SO}\left(1640.16 \mathrm{~cm}^{-1}\right.$, $1740.44 \mathrm{~cm}^{-1}$ ) and CO $\left(1643.05 \mathrm{~cm}^{-1}, 1741.41 \mathrm{~cm}^{-1}\right)$. A wavenumber around $2925 \mathrm{~cm}^{-1}$ (assigned to CO stretching) appeared at $2921.63 \mathrm{~cm}^{-1}$ for EVOO, at $2922.59 \mathrm{~cm}^{-1}$ for SO and at $2921.63 \mathrm{~cm}^{-1}$ for CO.

From Table (5), it was observed a shift of band around $2925 \mathrm{~cm}^{-1}$, this band is attributed to the symmetric stretching vibration of the aliphatic $\mathrm{CH}_{2}$ group which primarily showed absorbance at $2921.63 \mathrm{~cm}^{-1}$ of $91.13 \%$ intensity (EVOO). The intensity of this band shifted from $91.13 \%$ in EVOO to $85.19 \%$ (SO $5 \%$ ), then to $79.72 \%$ (SO $15 \%$ ) with band shift to $2922.59 \mathrm{~cm}^{-1}$ and $4.26 \%$ (SO $25 \%$ ) with band shift to $2924.52 \mathrm{~cm}^{-1}$, and the band shifted to $2922.59 \mathrm{~cm}^{-1}$ with intensity $96.04 \%$ for pure SO. This peak was important to detect the concentration of 5\% adulterant SO in EVOO.

Also, a very strong band of the $\mathrm{C}=\mathrm{O}$ stretching band of the ester group located at $1743.33 \mathrm{~cm}^{-1}$ in EVOO with intensity $92.30 \%$ shifted to $1742.37 \mathrm{~cm}^{-1}$ of intensity $86.88 \%$ (in SO 5\%), then it appears at $1743.33 \mathrm{~cm}^{-1}$ of intensity $83.09 \%$ (in SO $15 \%$ ), then it appears at $1745.26 \mathrm{~cm}^{-1}$ of intensity $9.61 \%$ (in SO $25 \%$ ), whereas, it appeared at $1740.44 \mathrm{~cm}^{-1}$ of intensity $95.54 \%$ (pure SO).

Table 5: FTIR spectral data of extra virgin olive oil in binary admixtures with sunflower oil at various concentrations of adulterant sunflower oil

\begin{tabular}{|c|c|c|c|c|c|c|c|c|c|}
\hline \multicolumn{2}{|c|}{ EVOO 100\% } & \multicolumn{2}{|c|}{ SO $100 \%$} & \multicolumn{2}{|c|}{ SO 5\% } & \multicolumn{2}{|c|}{ SO 15\% } & \multicolumn{2}{|c|}{ SO 25\% } \\
\hline $\mathbf{c m}^{-1}$ & $\% \mathrm{~T}$ & $\mathrm{~cm}^{-1}$ & $\% \mathrm{~T}$ & $\mathbf{c m}^{-1}$ & $\% \mathrm{~T}$ & $\mathbf{c m}^{-1}$ & $\% \mathrm{~T}$ & $\mathrm{~cm}^{-1}$ & $\% \mathrm{~T}$ \\
\hline 3467.38 & 96.97 & 3457.74 & 92.65 & 3457.74 & 91.04 & 3466.42 & 92.38 & 3472.2 & 90.83 \\
\hline 2921.63 & 91.13 & 2922.59 & 96.04 & 2921.63 & 85.19 & 2922.59 & 79.72 & 2924.52 & 4.26 \\
\hline \multirow[t]{2}{*}{2865.06} & 92.53 & 2855.1 & 96.63 & 2856.06 & 86.62 & 2856.06 & 81.69 & 2858.95 & 7.10 \\
\hline & & & & 2674.78 & 92.52 & 2677.68 & 92.15 & 2678.64 & 88.80 \\
\hline 2368.16 & 102.92 & 2376.84 & 98.80 & 2372.01 & 92.48 & 2366.23 & 93.49 & 2344.05 & 99.76 \\
\hline 2039.35 & 102.95 & & & & & 2058.64 & 95.01 & 2033.57 & 100.07 \\
\hline 1743.33 & 92.30 & 1740.44 & 95.54 & 1742.37 & 86.88 & 1743.33 & 83.09 & 1745.26 & 9.61 \\
\hline 1654.62 & 100.30 & 1640.16 & 95.40 & 1648.84 & 92.48 & 1654.62 & 93.10 & 1658.48 & 84.74 \\
\hline 1454.06 & 94.40 & 1450.21 & 95.84 & 1453.1 & 89.42 & 1454.06 & 86.83 & 1455.03 & 23.15 \\
\hline 1367.28 & 95.72 & 1376.93 & 95.88 & 1366.32 & 90.62 & 1366.32 & 88.66 & 1366.32 & 37.07 \\
\hline 1234.22 & 94.65 & & & & & 1232.29 & 87.27 & 1233.25 & 21.97 \\
\hline \multirow[t]{2}{*}{1160.94} & 93.02 & 1164.79 & 95.48 & 1159.01 & 87.84 & 1158.04 & 84.94 & 1162.87 & 9.94 \\
\hline & & 1021.12 & 96.37 & & & & & & \\
\hline 719.318 & 96.71 & & & 718.35 & 92.38 & 718.354 & 89.50 & 721.247 & 42.21 \\
\hline \multirow[t]{2}{*}{596.861} & 101.82 & 609.39 & 95.82 & & & 594.93 & 94.60 & 593.96 & 86.53 \\
\hline & & 434.869 & 97.71 & & & 443.54 & 96.11 & 456.082 & 92.11 \\
\hline
\end{tabular}




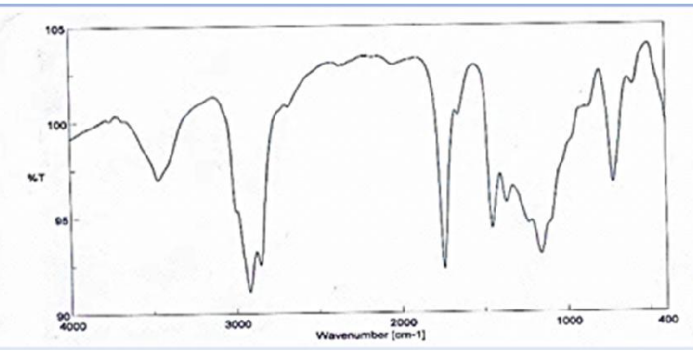

Fig. 1: FTIR of $100 \%$ extra virgin olive oil

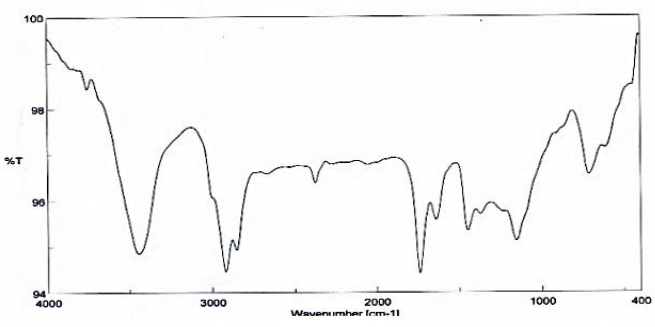

Fig. 3: FTIR of $100 \%$ corn oil

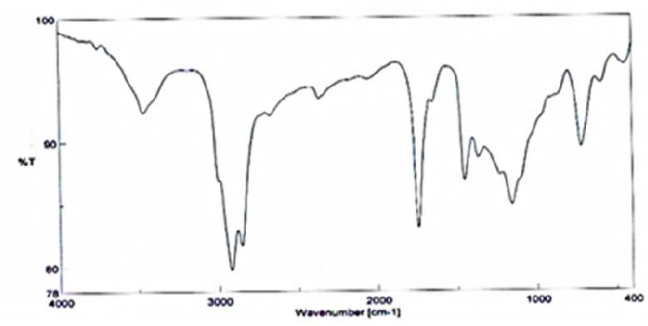

Fig. 5: FTIR of $15 \%$ sunflower oil

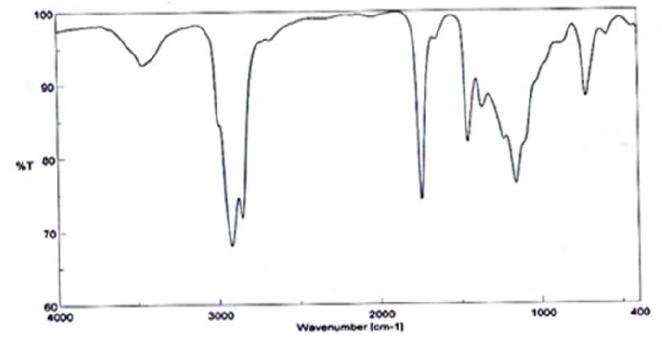

Fig. 7: FTIR of 5\% corn oil

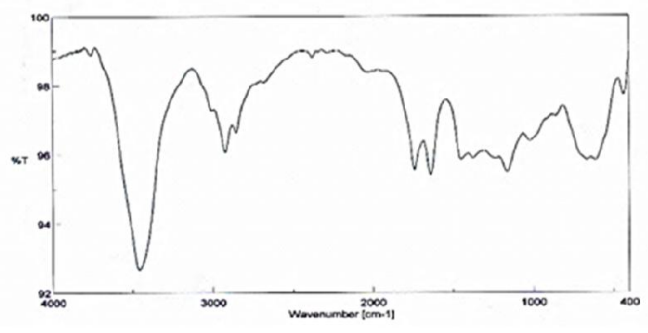

Fig. 2: FTIR of $100 \%$ sunflower oil

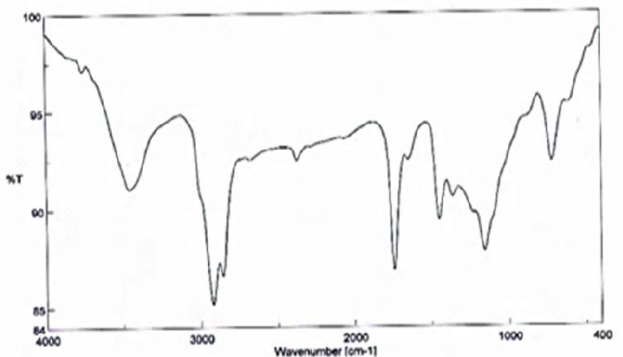

Fig. 4: FTIR of 5\% sunflower oil

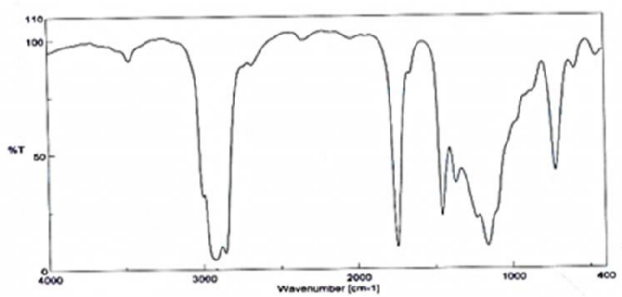

Fig. 6: FTIR of $25 \%$ sunflower oil

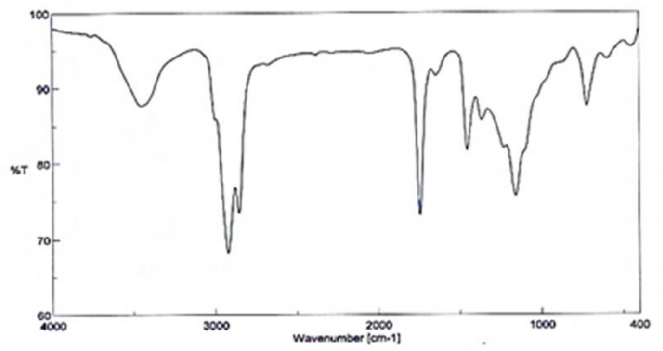

Fig. 8: FTIR of $15 \%$ corn oil

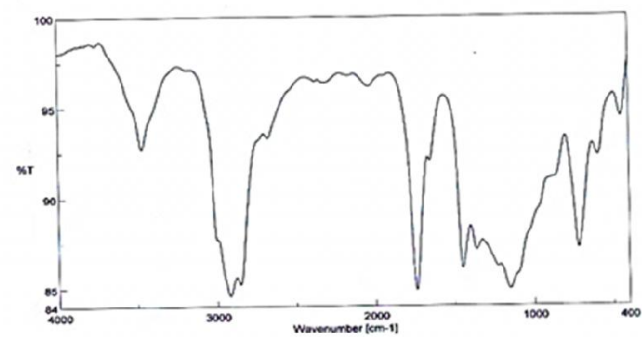

Fig. 9: FTIR of $25 \%$ corn oil

From Table (6), it was observed a shift of the intensity of the absorption band around $2925 \mathrm{~cm}^{-1}$, this band is attributed to the symmetric stretching vibration of the aliphatic $\mathrm{CH}_{2}$ group which primarily showed absorbance at $2921.63 \mathrm{~cm}^{-1}$ of $91.13 \%$ intensity in case of extra virgin olive oil. The intensity of this band shifted from $91.13 \%$ in EVOO to $68.08 \%$ (for CO 5\%) with band shift to $2922.59 \mathrm{~cm}^{-1}$, then to $68.13 \%$ (for CO $15 \%$ ) with band shift to $2923.56 \mathrm{~cm}^{-1}$ and $84.57 \%$ (for CO $25 \%$ ) with band at 
$2921.63 \mathrm{~cm}^{-1}$, and the band at $2921.63 \mathrm{~cm}^{-1}$ with intensity $94.45 \%$ for pure CO. This peak around 2920 $\mathrm{cm}^{-1}$ was important to detect concentration of $5 \%$ adulterant $\mathrm{CO}$ in EVOO.

Also, a very strong band of the $\mathrm{C}=\mathrm{O}$ stretching band of the ester group located at $1743.33 \mathrm{~cm}^{-1}$ in EVOO with intensity $92.30 \%$ shifted to $1744.3 \mathrm{~cm}^{-1}$ of intensity $74.23 \%$ (in CO $5 \%$ ), then it appears at $1744.3 \mathrm{~cm}^{-1}$ of intensity $73.19 \%$ (in CO $15 \%$ ), then it appears at $1742.37 \mathrm{~cm}^{-1}$ of intensity $84.87 \%$ (in CO $25 \%$ ), whereas, it appeared at $1741.41 \mathrm{~cm}^{-1}$ of intensity $94.41 \%$ (pure $\mathrm{CO}$ ).

Table 6: FTIR spectral data of extra virgin olive oil in binary admixtures with corn oil at various concentrations of adulterant corn oil

\begin{tabular}{llllllllll}
\hline \multicolumn{2}{c}{$\begin{array}{c}\text { EVOO 100\% } \\
\mathbf{c m}^{-\mathbf{1}}\end{array}$} & $\mathbf{\%} \mathbf{T}$ & \multicolumn{2}{c}{$\mathbf{C O} \mathbf{1 0 0} \%$} & \multicolumn{2}{c}{$\mathbf{C O ~ 5 \%}$} & \multicolumn{2}{c}{$\mathbf{C O} \mathbf{1 5 \%}$} & \multicolumn{2}{c}{$\mathbf{C O ~ 2 5 \%}$} \\
\hline 3467.38 & 96.97 & 3445.21 & 94.84 & 3465.46 & 92.75 & 3450.99 & 87.70 & 3470.28 & 98.45 \\
$\mathbf{2 9 2 1 . 6 3}$ & $\mathbf{9 1 . 1 3}$ & $\mathbf{2 9 2 1 . 6 3}$ & $\mathbf{9 4 . 4 5}$ & $\mathbf{2 9 2 2 . 5 9}$ & $\mathbf{6 8 . 0 8}$ & $\mathbf{2 9 2 3 . 5 6}$ & $\mathbf{6 8 . 1 3}$ & $\mathbf{2 9 2 1 . 6 3}$ & $\mathbf{8 4 . 5 7}$ \\
2865.06 & 92.53 & 2855.1 & 94.92 & 2857.02 & 71.82 & 2856.06 & 73.44 & 2856.06 & 85.22 \\
2368.16 & 102.92 & 2373.94 & 96.38 & & & 2379.73 & 94.40 & 2308.37 & 96.37 \\
2039.35 & 102.95 & & & 2040.32 & 99.19 & 2051.89 & 94.57 & 2036.46 & 96.12 \\
$\mathbf{1 7 4 3 . 3 3}$ & $\mathbf{9 2 . 3 0}$ & $\mathbf{1 7 4 1 . 4 1}$ & $\mathbf{9 4 . 4 1}$ & $\mathbf{1 7 4 4 . 3}$ & $\mathbf{7 4 . 2 3}$ & $\mathbf{1 7 4 4 . 3}$ & $\mathbf{7 3 . 1 9}$ & $\mathbf{1 7 4 2 . 3 7}$ & $\mathbf{8 4 . 8 7}$ \\
1654.62 & 100.30 & 1643.05 & 95.57 & 1653.66 & 96.12 & 1644.98 & 91.62 & 1655.59 & 92.00 \\
1454.06 & 94.40 & 1451.17 & 95.33 & 1455.03 & 82.08 & 1455.99 & 81.70 & 1453.1 & 86.04 \\
1367.28 & 95.72 & & & 1366.32 & 86.70 & 1366.32 & 85.75 & 1365.35 & 87.09 \\
1234.22 & 94.65 & & & 1233.25 & 82.38 & 1233.25 & 82.02 & & \\
1160.94 & 93.02 & 1157.08 & 95.12 & 1159.97 & 76.38 & 1160.94 & 75.66 & 1156.12 & 84.91 \\
719.318 & 96.71 & 712.56 & 96.54 & 720.282 & 88.12 & 720.282 & 87.54 & 719.318 & 87.17 \\
596.861 & 101.82 & & & 593.968 & 96.60 & 594.93 & 93.79 & 594.932 & 92.26 \\
& & & & 429.084 & 97.71 & 444.512 & 95.43 & 450.297 & 94.41 \\
\hline
\end{tabular}

\section{Conclusion}

The present work successfully achieved its aim included in the applications of UV and FTIR spectroscopy as rapid, cheap, nondestructive, authenticity measuring tools to assess the adulteration of extra virgin olive oil with other edible oils such as corn and sunflower oils in detection limit $15 \%$ for UV and 5\% for FTIR. Library searching in the FTIR region is a well established and powerful way which was used in comparison and matching of measured spectra. Adulteration of extra virgin olive oil with adulterant sunflower and corn oils could be detected at $15 \%$ of adulterant oil added using $\Delta \mathrm{K}$ values obtained from UV characteristics, and this is the lowest detectable value in adulterated extra virgin olive oil. Using FTIR spectroscopy via measuring the changes in a band around $2925 \mathrm{~cm}^{-1}$ along with its intensity (assigned to $\mathrm{C}-\mathrm{O}$ stretching and symmetric stretching vibration of the aliphatic $\mathrm{CH}_{2}$ group) along with a very strong band along with its intensity located at $1743.33 \mathrm{~cm}^{-1}$ (assigned to the $\mathrm{C}=\mathrm{O}$ stretching band of the ester group), these were important to detect concentrations of $5 \%$ of adulterant oils; $\mathrm{SO}$ and $\mathrm{CO}$ in EVOO.

In conclusion, UV spectroscopy in combination with FTIR spectroscopy offered effective tools for authentication of EVOO against the adulteration practice.

\section{References}

AlHanash, H.B., R.G. Elhabishi, W. Elhrari and R.A.M. Issa, 2018. The Use of Fourier-Transform Infrared Spectroscopy to Assess Libyan Virgin Olive Oil Adulteration with Corn and Sun Flower Oils. Academic Journal of Chemistry, Vol. 3, Issue. 5, pp: 40-45, 2018.

Allam, M.A., and S.F. Hamed, 2007. Application of FTIR spectroscopy in the assessment of olive oil adulteration. J. Applied Sciences Research, 3: (2), 102-108.

Almoselhy Rania, I.M., Allam Magda, H., A.A. Kotb and A. A. El-Sharkawy, 2009. Detection of Adulteration of Extra Virgin Olive Oil using FTIR Spectral Data. Research Bulletin, Ain Shams Univ., 2009. No.13, 2009 - 12 pages.

Bajoub, A., A. Bendini, A. Fernández-Gutiérrez and A. Carrasco-Pancorbo, 2018. Olive oil authentication: A comparative analysis of regulatory frameworks with especial emphasis on quality and authenticity indices, and recent analytical techniques developed for their assessment. 
A review, Critical Reviews in Food Science and Nutrition, 58:5, 832-857, DOI: 10.1080 $/ 10408398.2016 .1225666$

Caponio, F., A. Leone, G. Squeo, A. Tamborrino and C. Summo, 2019. Innovative technologies in virgin olive oil extraction process: influence on volatile compounds and organoleptic characteristics. J Sci Food Agric., 99: 5594-5600.

Codex Alimentarius Standard, 2013. Codex standard for olive oils and olive-pomace oils. CODEX STAN 33-1981/ Adopted in 1981. Revision: 1989, 2003, 2015. Amendment: 2009, 2013.

Commission Implementing Regulation (EU) No 1348/2013 of 16 December 2013 amending Regulation (EEC) No 2568/91 on the characteristics of olive oil and olive-residue oil and on the relevant methods of analysis (2013). Off. J. EU, L, 338, 31-67.

Gómez-Caravaca, A.M., R.M. Maggio, L. Cerretani, 2016. Chemometric applications to assess quality and critical parameters of Virgin and Extra-Virgin Olive Oil. A Review, Analytica Chimica Acta (2016), doi: 10.1016/j.aca.2016.01.025.

Gonçalves, R.P., P. H. Março and P. Valderrama, 2014. Thermal edible oil evaluation by UV-Vis spectroscopy and chemometrics. Food Chemistry 163 (2014) 83-86.

Guillén, M.D. and N. Cabo, 1999. Usefulness of the frequencies of some Fourier transform infrared spectroscopic bands for evaluating the composition of edible oil mixtures. Fett/Lipid 101 (1999), Nr. 2, S. 71-76.

IOC, 2019a. Spectrophotometric investigation in the ultraviolet. COI/T.20/Doc. No 19/Rev. 5, International Olive Council.

IOC, 2019b. Trade standard applying to olive oils and olive-pomace oils. COI/T.15/NC No 3/Rev. 14, International Olive Council.

Kates, N., 1972. Technique of Lipidology, Elsevier Publishing Co. Inc.

Lerma-García, M.J., G. Ramis-Ramos, J.M. Herrero-Martínez and E.F. Simó-Alfonso, 2010. Authentication of extra virgin olive oils by Fourier-transform infrared spectroscopy. Food Chemistry, $118: 78-83$.

Monika, G.D., P. Przychodzen, F. Cappello, A. Kuban-Jankowska, A. M. Gammazza, N. Knap, M. Wozniak, and M. Gorska-Ponikowska, 2018. Potential Health Benefits of Olive Oil and Plant Polyphenols. Int. J. Mol. Sci., 2018, 19, 547.

Nurwahidah, A.T., S. Rumiyati Riyanto, A.F. Nurrulhidayah, K. Betania and A. Rohman, 2019. Fourier Transform Infrared Spectroscopy (FTIR) coupled with multivariate calibration and discriminant analysis for authentication of extra virgin olive oil from rambutan seed fat. Food Research 3 (6): 727 - 733 (December 2019).

Rohman, A., F.M. Yusof and Y.B. Che Man, 2014. The Use of FTIR Spectroscopy and Chemometrics for Rapid Authentication of Extra Virgin Olive Oil. J Am Oil Chem Soc (2014) 91:207-213. 